\title{
Efficient subtraction of insect rRNA prior to transcriptome analysis of Wolbachia-Drosophila lateral gene transfer
}

Nikhil Kumar ${ }^{1}$, Todd Creasy ${ }^{1}$, Yezhou Sun ${ }^{1}$, Melissa Flowers ${ }^{1}$, Luke J Tallon ${ }^{1}$ and Julie C Dunning Hotopp ${ }^{1,2^{*}}$

\begin{abstract}
Background: Numerous methods exist for enriching bacterial or mammalian mRNA prior to transcriptome experiments. Yet there persists a need for methods to enrich for mRNA in non-mammalian animal systems. For example, insects contain many important and interesting obligate intracellular bacteria, including endosymbionts and vector-borne pathogens. Such obligate intracellular bacteria are difficult to study by traditional methods. Therefore, genomics has greatly increased our understanding of these bacteria. Efficient subtraction methods are needed for removing both bacteria and insect rRNA in these systems to enable transcriptome-based studies.
\end{abstract}

Findings: A method is described that efficiently removes $>95 \%$ of insect rRNA from total RNA samples, as determined by microfluidics and transcriptome sequencing. This subtraction yielded a 6.2-fold increase in mRNA abundance. Such a host rRNA-depletion strategy, in combination with bacterial rRNA depletion, is necessary to analyze transcription of obligate intracellular bacteria. Here, transcripts were identified that arise from a lateral gene transfer of an entire Wolbachia bacterial genome into a Drosophila ananassae chromosome. In this case, an rRNA depletion strategy is preferred over polyA-based enrichment since transcripts arising from bacteria-to-animal lateral gene transfer may not be poly-adenylated.

Conclusions: This enrichment method yields a significant increase in mRNA abundance when poly-A selection is not suitable. It can be used in combination with bacterial rRNA subtraction to enable experiments to simultaneously measure bacteria and insect mRNA in vector and endosymbiont biology experiments.

Keywords: Wolbachia, Drosophila ananassae, RNASeq, Transcriptomics, Lateral gene transfer, Horizontal gene transfer, Endosymbiont, Insect vector

\section{Findings}

\section{Background}

Many interesting bacteria form intimate, obligate relationships with eukaryotes. These bacteria include endosymbionts and obligate intracellular pathogens. These microbes can be difficult to research as they cannot be cultured, easily manipulated, or genetically transformed. Therefore, genomics techniques have significantly advanced the study of these organisms. Because of the obvious potential health impacts, many techniques and tools have been developed to research such bacteria that

\footnotetext{
* Correspondence: jdhotopp@som.umaryland.edu

${ }^{1}$ Institute for Genome Sciences, University of Maryland School of Medicine, Baltimore, MD 21201, USA

${ }^{2}$ Department of Microbiology and Immunology, University of Maryland School of Medicine, Baltimore, MD 21201, USA
}

interact with humans. For example, the Ribo-Zero rRNA removal kit for human/mouse/rat (Epicentre, Madison, WI, USA) can facilitate transcriptome analysis of intracellular pathogens of humans. However, for non-mammalian systems including bacteria/vector systems, a void still exists. Previously, the MICROBEnrich insect/C. elegans module (Ambion, Austin, TX, USA) was used for this purpose, but it is no longer available. Therefore, we sought to investigate if Epicentre's Ribo-Zero rRNA removal kit designed for humans and rodents would efficiently remove rRNA from insect samples. Drosophila ananassae is a fruit fly that can be naturally infected with a Wolbachia endosymbiont [1,2]. In addition, some Wolbachia-colonized lines have an entire Wolbachia genome transferred to a fly nuclear chromosome [3]. Transcripts from nuclear Wolbachia transfers (nuwts) are of particular interest, as

\section{Biomed Central}



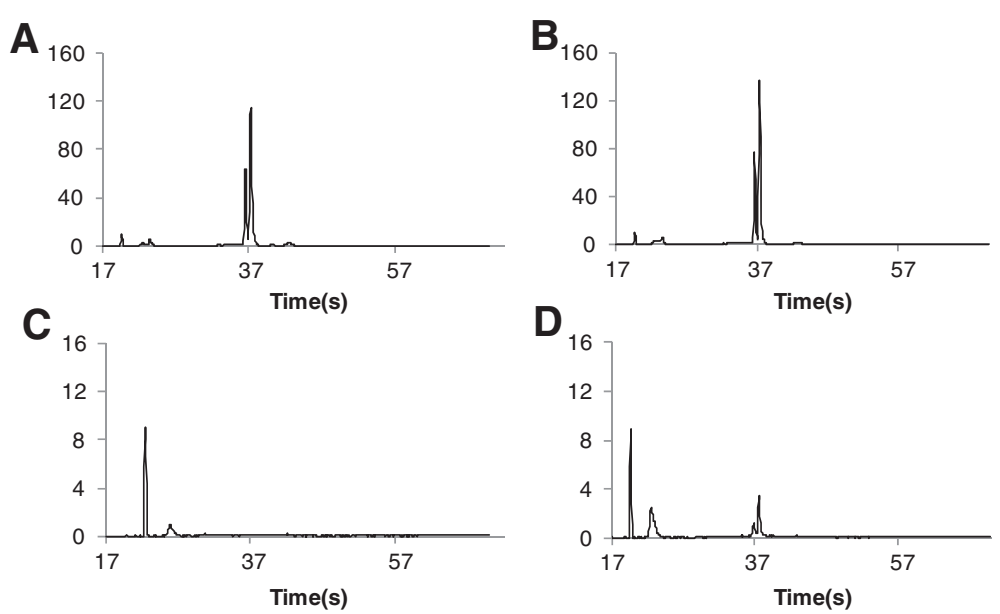

Figure 1 Bioanalyzer analysis of Ribo-Zero-subtracted RNA. The subtraction of rRNA using the Ribo-Zero human/mouse/rat reagents was tested on total RNA from Drosophila ananassae with (panels $\mathbf{A}$ and $\mathbf{C}$ ) and without (panels $\mathbf{B}$ and $\mathbf{D}$ ) the presence of its Wolbachia endosymbiont. The total RNA prior to Ribo-Zero subtraction (panels $\mathbf{A}$ and $\mathbf{B}$ ) is compared to RNA after Ribo-Zero subtraction (panels $\mathbf{C}$ and $\mathbf{D}$ ). The starting amount of RNA prior to subtraction for panels $\mathbf{C}$ and $\mathbf{D}$ was equivalent to the amount shown in panels $\mathbf{A}$ and $\mathbf{B}$.

they can be used to elucidate functions for the nuwts. Previously, we identified 28 nuwt transcripts ( 2\% of the Wolbachia genome) in adult flies, albeit at low levels [3]. Since these transcripts might not be polyadenylated, polyA enrichment of total RNA is not a suitable technique for obtaining mRNA prior to RNASeq. Therefore, Ribo-Zero was tested as a suitable alternative.

\section{RNA isolation, mRNA enrichment, and transcriptome sequencing}

Total RNA was isolated with TRIzol, as previously described [3], from 50-85 freshly laid eggs of wild-type D. ananassae In(3R)A (Stock No. 14024-0371.13) that is infected with the Wolbachia endosymbiont wAna [2] and from a tetracycline-treated (Wolbachia-cured) line of this fly. Both lines are tested regularly by fluorescence in situ hybridization to confirm the presence or absence of a Wolbachia infection, respectively. The samples were enriched for mRNA using the Ribo-Zero rRNA removal kit for human/mouse/rat (Epicentre, Madison, WI, USA), following the manufacturer's protocol.

\section{Assessment of rRNA removal by microfluidics}

Comparison of the original and Ribo-Zero-subtracted samples on a Bioanalyzer (Agilent, Santa Clara, CA, USA) illustrates the efficient removal of the majority of the rRNA (Figure 1) and the loss of $>97 \%$ of the RNA, as assessed by integrating the area under the rRNA peaks. In $D$. ananassae, as well as in many insects, the $28 \mathrm{~S}$ rRNA is naturally cleaved resulting in two peaks that are unusually close together on the Bioanalyzer when compared to other eukaryotic or bacterial rRNA samples. Importantly, the Ribo-Zero subtraction efficiently removed both halves of the cleaved rRNA.

\section{Assessment of rRNA removal by transcriptome sequencing}

While the Bioanalyzer is highly sensitive at detecting intact rRNA, degraded rRNA can go undetected. Therefore,

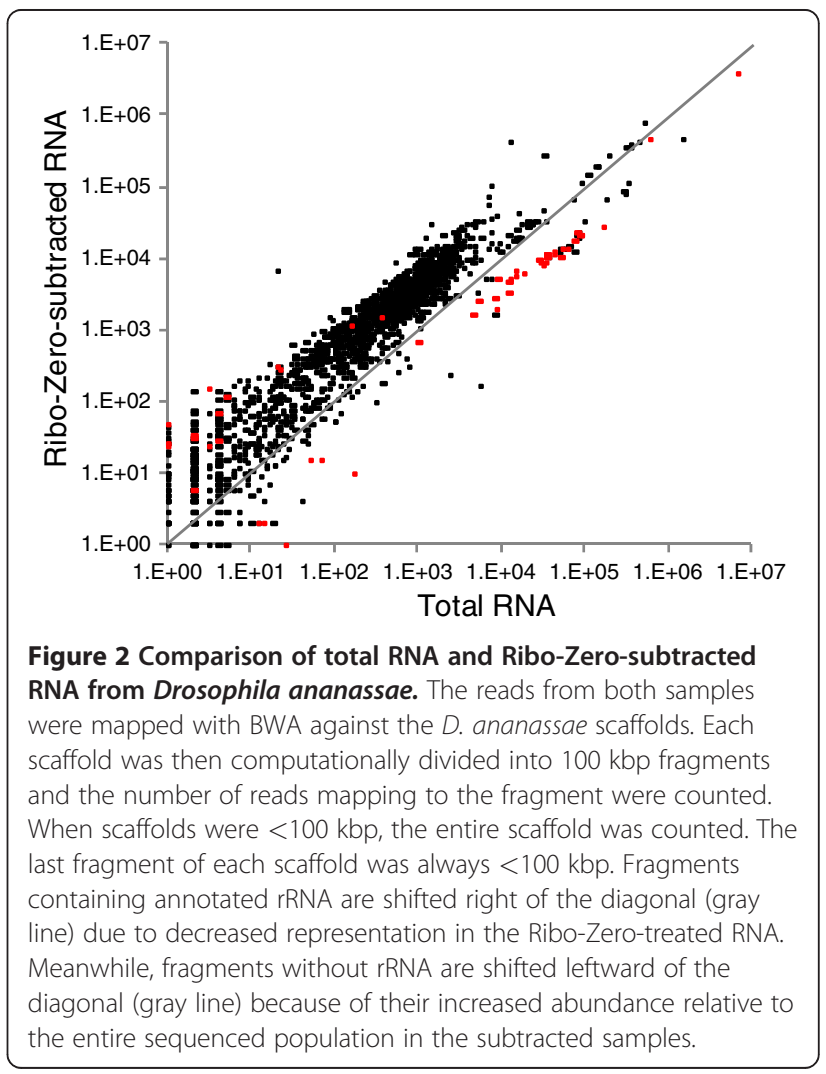




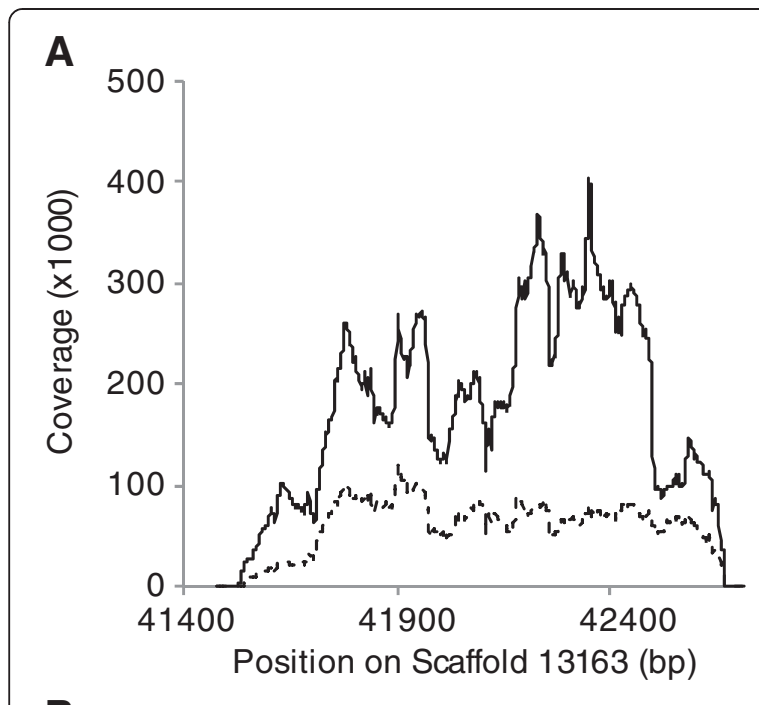

B

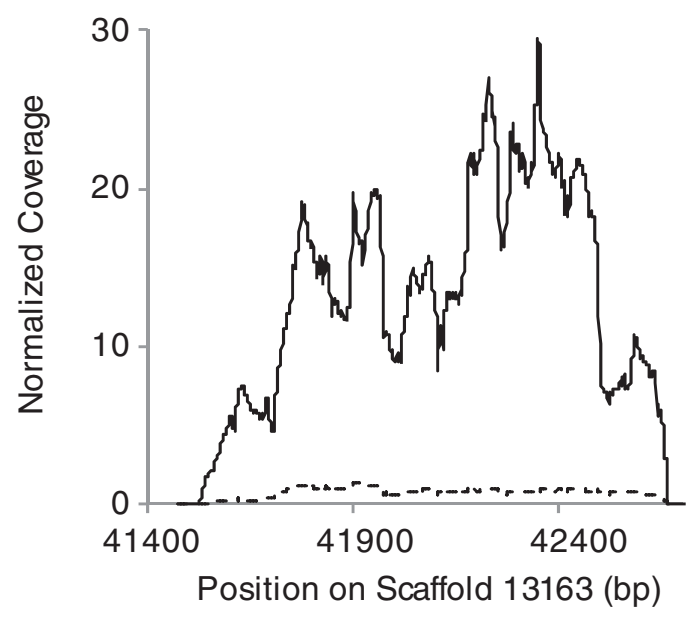

C

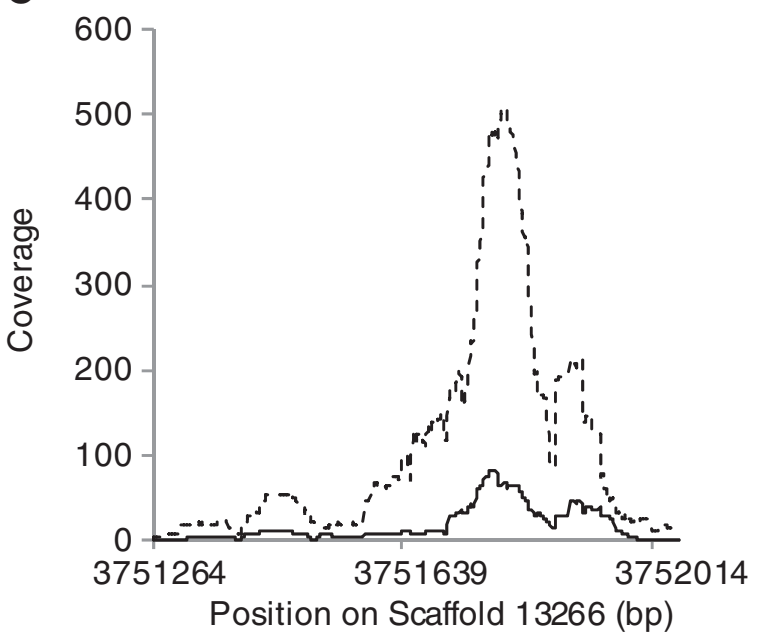

Figure 3

we also sought to examine RNASeq results to ensure even subtraction across the rRNA. Paired-end libraries (Illumina, San Diego, CA, USA) were constructed using
Figure 3 Coverage of the 18S rRNA and actin genes. The coverage of reads across the 18S rRNA (Panel A) and actin (Panel C) genes was determined by mpileup in samtools and compared between total RNA (solid line) and Ribo-Zero-subtracted RNA (dashed line). For the $18 \mathrm{~S}$ rRNA, the Ribo-Zero-subtracted sample contained $66 \%$ fewer rRNA reads when compared to the total RNA sample. When the $18 \mathrm{~S}$ rRNA results are normalized relative to actin, a 95\% reduction in the rRNA is seen (Panel B). The 18S rRNA is only partially sequenced in the reference genome with a gap in the scaffold to the immediate right of this region.

the two pools of RNA for the cured line (Figure 1B and 1D) using the standard protocol starting immediately after the poly-A selection step. Half of a channel of 72-bp reads was obtained on a GAIIx (Illumina, San Diego, CA, USA) for each library. The sequencing reads were mapped against the reference $D$. ananassae assembly, using the default parameters for BWA [4], yielding 15.8 million and 15.0 million mapped reads from total RNA and the Ribo-Zero-subtracted sample, respectively. The reads for each $100 \mathrm{kbp}$ window across the genome are plotted, comparing the subtracted sample from the total RNA (Figure 2). In this subtraction, and unlike the subtraction with the RNA from uncured specimens, the Bioanalyzer revealed that only $98 \%$ of rRNA was removed. Therefore, and as expected, a significant amount of rRNA is still present, as illustrated by the red dots representing fragments with at least a portion of an rRNA. However, the results are consistent with removal of $>90 \%$ of the rRNA with a shift leftward of the majority of $100 \mathrm{kbp}$ fragments due to enrichment for mRNA and separation of the rRNA from the mRNA by approximately one order of magnitude.

While the overall trend is clear, specific points may not reflect this trend. For example, the $100 \mathrm{kbp}$ region containing the $18 \mathrm{~S}$ rRNA is the red point in the upper right corner that appears unshifted from the diagonal. In the total RNA, 2,936,794 reads mapped to the 18S rRNA fragment (41,477-43,516 bp on scaffold 13163; GenBank CH902719.1); in the subtracted sample 980,094 reads (66\%) were sequenced (Figure 3A). This would be sufficient to offset the point from diagonal except that an additional $\sim 3$ million reads map in this $100 \mathrm{kbp}$ fragment and outside the $18 \mathrm{~S}$ rRNA. These reads prevent the full shift of this point relative to the diagonal that would be realized should only the 18S rRNA be present in this fragment.

While there is a $66 \%$ difference in the raw number of $18 \mathrm{~S}$ rRNA reads between the two samples (Figure 3A), this does not fully capture the Ribo-Zero subtraction. Subtraction of rRNA means that significantly more reads are obtained from the remaining RNA species in the subtracted sample, as seen in the 6.2-fold increase in signal for actin in the subtracted sample (Figure 3C). When the rRNA results for each sample are normalized to the 
Table 1 Wolbachia genes represented in reads and arising from nuwts

\begin{tabular}{|c|c|c|c|c|c|c|c|}
\hline \multirow[b]{2}{*}{5 '-end } & \multirow[b]{2}{*}{ 3'-end } & \multirow[b]{2}{*}{ Locus } & \multirow[b]{2}{*}{ Name } & \multicolumn{2}{|c|}{ Pairs of Reads } & \multicolumn{2}{|c|}{ Unpaired Reads } \\
\hline & & & & $\begin{array}{l}\text { Total } \\
\text { RNA }\end{array}$ & $\begin{array}{l}\text { Ribo-Zero- } \\
\text { subtracted }\end{array}$ & $\begin{array}{l}\text { Total } \\
\text { RNA }\end{array}$ & $\begin{array}{l}\text { Ribo-Zero- } \\
\text { subtracted }\end{array}$ \\
\hline 8305 & 10323 & WRi_000090 & type IV secretion system protein VirD4 & 0 & $2^{*}$ & 0 & 0 \\
\hline 20819 & 29332 & WRi_000230 & DNA-directed RNA polymerase, beta subunit & 0 & 1 & 0 & 0 \\
\hline 37758 & 35593 & WRi_000280 & ankyrin repeat domain protein & 0 & 1 & 0 & 0 \\
\hline 71725 & 72540 & WRi_000680 & hypothetical protein & 1 & 0 & 0 & 0 \\
\hline 188532 & 191277 & WRi_r01850 & $23 \mathrm{~S}$ ribosomal RNA & 0 & 0 & $1 \%$ & 0 \\
\hline 458087 & 457221 & WRi_004260 & 4-diphosphocytidyl-2 C-methyl-D-erythritol kinase & 0 & 1 & 0 & 0 \\
\hline 663596 & 664402 & WRi_006160 & hypothetical protein & 1 & 0 & 0 & 0 \\
\hline 687296 & 685893 & WRi_006360 & transcription termination factor Rho & 0 & 1 & 0 & 0 \\
\hline 804498 & 806600 & WRi_007420 & hypothetical protein & $3^{*}$ & 0 & 0 & 0 \\
\hline 832611 & 833609 & WRi_007700 & tryptophanyl-tRNA synthetase & 0 & 1 & 0 & 0 \\
\hline 938372 & 936990 & WRi_008690 & transposase & $2^{*,+}$ & 0 & & \\
\hline 1044899 & 1045516 & WRi_009720 & lipoyltransferase & 0 & 0 & 1 & 0 \\
\hline 1139870 & 1138959 & WRi_010540 & transcriptional regulator, putative & 0 & $1+$ & 0 & 0 \\
\hline 1289969 & 1291473 & WRi_r11990 & $16 \mathrm{~S}$ ribosomal RNA & 0 & 0 & $1 \%$ & $2 \%$ \\
\hline 1334777 & 1336324 & WRi_012430 & penicillin-binding protein & 1 & 0 & 0 & 0 \\
\hline 1336345 & 1337274 & WRi_012440 & 4-hydroxy-3-methylbut-2-enyl diphosphate reductase & 1 & 0 & 0 & 0 \\
\hline 1436571 & 1435714 & WRi_013460 & 4-hydroxybenzoate octaprenyltransferase & 0 & 1 & 0 & 0 \\
\hline
\end{tabular}

"These are duplicate paired end reads; upon manually removing duplicates this reduces to one. However, these reads were not removed when analyzed with the duplicate analysis tool Picard.

${ }^{t}$ These reads are not unique with respect to the reference genome.

these reads likely arise from non-Wolbachia bacterial contaminants on the eggs.

number of reads in actin, a 95\% reduction of rRNA is observed (Figure 3B). This is consistent with the 98\% reduction determined by integrating the area under the peak on the Bioanalyzer.

Not only does the subtraction increase the signal for genes as shown above with actin, it also increases the number of genes that can be analyzed. While 8,888 transcripts had at least a single read mapping in the subtracted sample, only 7,629 transcripts had a single read mapping in the unsubtracted sample. Yet, a single read is not very informative when examining differential expression, and instead, a minimum number of reads/transcript may be required for a differential expression analysis. Standards for this minimum have not been established to our knowledge. But if one required 100 reads/transcript, the subtracted sample would have 3,677 transcripts that could be analyzed while the unsubtracted sample would only have 1,047 transcripts.

\section{Detection of transcripts arising from bacteria-to-animal lateral gene transfer}

While the rRNA was sufficiently removed from the studied samples, only a few reads (19 and 20 reads from the total RNA and the Ribo-Zero-subtracted
RNA, respectively) arose from transcripts of nuwts as identified by mapping with BWA [4] against the wRi reference genome [5] (Table 1). No transcripts were identified with more than one mapped read after duplicate removal. This low abundance of reads is not sufficient for analysis of the nuwt transcriptome. There was no overlap in the reads found between the two samples with the exception of reads that likely arose from nonWolbachia bacterial contaminants on the surface of the eggs, further suggesting the stochastic detection of these low abundance transcripts.

This low abundance of transcription mirrors previous findings that transcription of nuwts is low [3]. In previous work, nuwts were found to be $10^{4}-10^{7}$ times less abundant than actin in adult flies [3], which is consistent with the RNASeq results presented here for eggs. This level of transcription may or may not be biologically relevant. Important tissue-, condition-, and/or stage-specific transcription cannot be ruled out. However the tissue, condition, and/or stage that should be examined are not immediately obvious in the absence of a phenotype.

\section{Conclusions}

The Ribo-Zero rRNA removal kit for human/mouse/rat efficiently removes $>98 \%$ of insect rRNA from total 
RNA samples, yielding a 6.2-fold increase in detection of mRNA transcripts. In this data, $3 \times$ as many transcripts can be evaluated in a differential gene expression analysis requiring at least 100 reads/transcript. Coupling this Ribo-Zero kit with ones designed for removal of bacterial rRNA would also easily reduce endosymbiont rRNA and allow the concomitant sequencing of host and bacterial mRNA from endosymbiont containing samples.

\section{Availability of supporting data}

The sequencing reads supporting the results of this article are available in the sequence read archive, SRA045520 at http://www.ncbi.nlm.nih.gov/sra?term=SRA045520.

\section{Abbreviations}

nuwt: Nuclear Wolbachia transfer.

\section{Competing interests}

The authors declare that they have no competing interests.

\section{Acknowledgements}

This work was supported by the NSF Microbial Sequencing Program (EF-0826732).

\section{Authors' contributions}

NK reared the flies including curing with tetracycline, isolated RNA for the experiments, performed the RNA subtractions, and drafted portions of the manuscript. TC, YS, and LJT analyzed the data and generated figures. MF and LJT were responsible for sequencing the RNA. JCDH conceived of the study, participated in its design and coordination, and drafted the manuscript. All authors read and approved the final manuscript.

Received: 31 January 2012 Accepted: 14 May 2012

Published: 14 May 2012

\section{References}

1. Bourtzis K, Nirgianaki A, Markakis G, Savakis C: Wolbachia infection and cytoplasmic incompatibility in Drosophila species. Genetics 1996, 144:1063-1073.

2. Salzberg SL, Dunning Hotopp JC, Delcher AL, Pop M, Smith DR, Eisen MB, Nelson WC: Serendipitous discovery of Wolbachia genomes in multiple Drosophila species. Genome Biol 2005, 6:R23.

3. Dunning Hotopp JC, Clark ME, Oliveira DC, Foster JM, Fischer P, Torres MC, Giebel JD, Kumar N, Ishmael N, Wang S, et al: Widespread lateral gene transfer from intracellular bacteria to multicellular eukaryotes. Science 2007, 317:1753-1756.

4. Li H, Durbin R: Fast and accurate short read alignment with BurrowsWheeler transform. Bioinformatics 2009, 25:1754-1760.

5. Klasson L, Westberg J, Sapountzis P, Naslund K, Lutnaes Y, Darby AC, Veneti Z, Chen $L$, Braig HR, Garrett R, et al: The mosaic genome structure of the Wolbachia wRi strain infecting Drosophila simulans. Proc Natl Acad Sci U S A 2009, 106:5725-5730.

\section{Submit your next manuscript to BioMed Central and take full advantage of:}

- Convenient online submission

- Thorough peer review

- No space constraints or color figure charges

- Immediate publication on acceptance

- Inclusion in PubMed, CAS, Scopus and Google Scholar

- Research which is freely available for redistribution 